\title{
DAS BERUFSHEER DER FRÜHEN UND HOHEN KAISERZEIT UND DIE VERARMUNG DER KAISERLICHEN ZENTRALE
}

\author{
ARMin Eich
}

Die folgenreichste Entscheidung,* die Augustus in seiner Herrschaftszeit traf, war die Einrichtung einer Berufsarmee von ca. 300000 Mann Sollstärke mit fixen Besoldungs- und Versorgungsansprüchen für die Mannschaftsdienstgrade und hohen, hierarchisch gestuften Salären für Unteroffiziere und Offiziere. ${ }^{1}$ Die Gesamtkosten, nur für Soldzahlungen und postdienstliche Versorgung, sind jüngst auf ca. 370 Millionen Sesterzen per annum geschätzt worden (andere Schätzungen bewegen sich in ähnlichen Größenordnungen). ${ }^{2}$ Für dieses Wagnis mochte sprechen, daß Augustus gigantische Mittel unter seine Kontrolle gebracht hatte: Er beherrschte die Geldemissionen und die Edelmetallvorräte des Imperiums nach Belieben, sein Patrimonium erstreckte sich über die gesamte Ökumene, und einige der reichsten Staaten der bekannten Welt waren im Laufe der Expansion der res publica Romana als steuerpflichtiges Untertanengebiet in das Imperium integriert worden. Auf der anderen Seite existierte offenkundig ein erheblicher sozialer Druck, die in den Bürgerkriegen entfesselten Militärklientelen politisch wieder einzubinden: Die Stiftung geregelter Versorgungsansprüche diente zweifellos auch der sozialen Pazifizierung und verarbeitete insofern die Erfahrung der vergangenen, von innerer Gewalt geprägten Jahrzehnte.

In den folgenden Ausführungen soll dennoch, d. h. obwohl die genannten Faktoren dem Sieger der ,Römischen Revolution' anscheinend Recht gaben, die These vertreten werden, daß die augusteische Entscheidung die ökonomischen Möglichkeiten des Imperiums überforderte oder, anders gewendet, daß sie die entscheidende Weichenstellung

\footnotetext{
* Meinem Bruder Peter Eich danke ich für viele Hinweise und Anregungen.

1 Die einschlägigen Daten und Quellen bei: K. Raaflaub, ,Die Militärreformen des Augustus und die politische Problematik des frühen Prinzipats', in G. Binder, Hrsg., Saeculum Augustum I (Darmstadt 1987), 246ff. Die Zahl 300000 wurde wohl erst nach Augustus erreicht. Genaue Daten für die Auxiliare fehlen.

${ }_{2}$ B. Campbell, War and Society in Imperial Rome (London u.a. 2002), 175.
} 
darstellte, die à la longue durée den Untergang des Imperiums katalysierte. Damit wenden sich die folgenden Zeilen explizit gegen die in der jüngeren Zeit populären Interpretationen, denen zufolge die Krisenphänomene des dritten Jahrhunderts und der Spätantike nur als (zeitlich oder regional) punktuell begrenzte Probleme zu deuten seien. ${ }^{3}$ An dieser Stelle kann die Argumentation nur unter dem Gesichtspunkt des Verhältnisses von Ausgabendruck und militärischen Konstellationen verfolgt werden. Dieser ist jedoch der entscheidende: Augustus hatte das von ihm geschaffene System unter das unerbittliche Diktat permanent anfallender (und steigender) Ausgaben gestellt. Die Mehrzahl der Untertanen arbeitete und handelte unter dem ständigen Druck, dem Staat die benötigten Ressourcen zur Verfügung zu stellen, die kaiserliche Zentrale war in ihren Handlungsmöglichkeiten immer stärker durch den kategorischen Imperativ eingeschränkt, die überlebensnotwendigen Mittel zu extrahieren. Im Westen kollabierte das System nach langer Agonie zu Beginn des fünften Jahrhunderts; ${ }^{4}$ im Osten bildete sich ein Rumpfimperium in einen Feudalstaat um. Die Frage, wie sich die stufenweise Verschärfung des Niedergangs auf verschiedenen gesellschaftlichen Feldern fühlbar machte, kann im vorgegebenen Rahmen nicht behandelt werden. Soviel nur sei angemerkt: Schon angesichts der Möglichkeiten und der Reichtümer, über die das Imperium verfügte, ist nicht damit zu rechnen, daß sich Krisenphänomene ubiquitär und in gleichmäßiger Manier in allen Sektoren nachweisen lassen. Aus diesem Grunde sollten einzelne archäologische Befunde, die beispielsweise im dritten Jahrhundert in der einen oder anderen Region lokale Prosperität erschließen lassen, nicht überbewertet werden. Um Illusionen bezüglich der allgemeinen Entwicklungstendenz zu vermeiden, sind bei Betrachtung des Imperium Romanum (wie bei anderen Gesellschaften) die Gesamtindikatoren zu betrachten. So hat etwa die quantitative Auswertung der mediterranen Wrackfunde ergeben, daß die Schiffahrtintensität von ihrem Höhepunkt in augusteischer Zeit in mehreren Stufen (in Gestalt eines Treppenkurvenverlaufs) auf ein Niveau abfiel, das im vierten Jahrhundert n. Chr. dem sechsten vorchristlichen

${ }^{3}$ Vgl. bspw. B. Meißner, ,Über Zweck und Anlaß von Diokletians Preisedikt ${ }^{6}$, Historia 49 (2000), 79ff.; Chr. Witschel, Krise - Rezession - Stagnation. Der Westen des römischen Reiches im 3. Fahrhundert n. Chr. (Frankfurt a.M. 1999); D. Rathbone, 'Monetisation, not Price-Inflation, in Third-century AD Egypt?', C.E. King, u.a. (Hrsgg.), Coin Finds and Coin Use in the Roman World, Kolloquium 1993 (Berlin 1996), $321 \mathrm{ff}$; K. Strobel, Das Imperium Romanum im 3. Fahrhundert - Modell einer historischen Krise? (Stuttgart 1993).

${ }_{4}^{4} \mathrm{Ph}$. Richardot, La fin de l'armée romaine (Paris 2001), 67ff.; 90. 
Jahrhundert entsprach. ${ }^{5}$ Einen analogen ,Gesamtindikator ${ }^{6}$ benennt Hans-Joachim Drexhage: „Der Rückgang der gesamten papyrologischen Überlieferung macht uns dramatisch deutlich, daß sich im 3. Jh. n. Chr. im römischen Ägypten insofern ein tiefer Wandel vollzogen hat, als ein Rückgang jeglicher, wirtschaftlicher Aktivitäten' konstatierbar ist. “6 Der bedeutendste dieser Gesamtindikatoren ist die wachsende ,Armut der kaiserlichen Zentrale', die sich angesichts der annähernden Monopolisierung der Edelmetallvorkommen durch den Staat fast paradox ausnimmt. Die Ursachen dieser Verarmung der Kaiser sind im wesentlichen wohl schon richtig gesehen worden. Doch eine etwas weitergehende Systematisierung der vorhandenen Beobachtungen ist möglich und (da die ,optimistische Interpretation' der Reichsgeschichte sie zu marginalisieren droht) sinnvoll. Diese Aufgabe möchte ich nun in Angriff nehmen.

\section{Symptome der Verarmung der kaiserlichen Zentrale}

Es ist in jüngerer Zeit, vor allem von Claude Domergue, ${ }^{7}$ darauf hingewiesen worden, daß die Förderung von Edelmetall im Imperium im Laufe des dritten Jahrhunderts an seine natürlichen Grenzen zu stoßen schien. Georges Depeyrot und Dominique Hollard ${ }^{8}$ haben ihre kliometrischen Untersuchungen daher auch unter das Motto der ,pénurie d'argent-métal' gestellt. Doch die ,natürlichen' Grenzen der Erzförderung waren relativ, sie bemaßen sich an den Bedürfnissen, die der römische Staat an den Bodenreichtum stellte. Im Mittelalter ist beständig Gold und Silber in großen Quantitäten aus europäischen Minen gefördert worden, die ,dakischen' Gruben gehören noch heute zu den reichsten der Welt. Das Problem der römischen Kaiser war, daß ihre Ansprüche die Fördergeschwindigkeit der Minen und den

\footnotetext{
${ }^{5}$ D. Gibbins, 'Shipwrecks and Hellenistic Trade', in Z.H. Archibald u.a., Hrsgg., Hellenistic Economies (London u.a. 2001), 273ff., 279.

${ }^{6}$ Preise, Mieten/Pachten, Kosten und Löhne im römischen Ägypten bis zum Regierungsantritt Diokletians (St. Katharinen 1991), 452f.

7 Les mines de la péninsule ibérique dans l'antiquité romaine (Rom 1990), 215ff.; J.C. Edmonson, 'Mining in the Later Roman Empire and beyond: Continuity or Disruption?', Fournal of Roman Studies 79 (1989), 84ff;; Chr. Howgego, 'The Supply and Use of Money in the Roman World', Fournal of Roman Studies 82 (1992), lff., 8.

8 'Pénurie d'argent-métal et crise monétaire au $\mathrm{III}^{\mathrm{e}}$ siècle après J.-C.', Histoire et Mesure 2 (1987), $57 \mathrm{ff}$.
} 
Rhythmus der Neuerschließungen bei weitem überstiegen. Der Nachschub, der den fiskalischen Ansprüchen in augusteischer Zeit genügt hatte, tat dies im dritten Jahrhundert nicht mehr. Von Phasenverschiebungen und Unterbrechungen im einzelnen abgesehen, gilt die Regel, daß seit Marcus die absolute Menge emittierten Silbers absank. ${ }^{9}$ Der steigende Ausgabendruck zwang die Zentrale dazu, die sinkende Gesamtsilbermenge in Pseudosilbermünzen zu legieren, deren absolute Anzahl im 3. Jh. bis zur Regierungszeit Aurelians beharrlich anstieg: Bei einer Indizierung von 100 in der Regierungszeit von Philippus Arabs ${ }^{10}$ erreicht die Indexzahl emittierter Münzen unter Aurelian einen Wert von $1000 .{ }^{11}$

Der Umstand, daß das Römische Reich abgesehen von den Hochkulturen jenseits des Euphrat bzw. Tigris alle Staaten, die Konkurrenzwährungen in Edelmetall hätten ausprägen können, gewaltsam integriert und damit neutralisiert hatte, gewährte der kaiserlichen Zentrale die Möglichkeit, (jedenfalls der Intention nach) den Wert der von ihr emittierten Münzen von dem Marktwert des in den Münzen enthaltenen Metalls abzukoppeln: Den Wert der Münze sollten staatliche Hoheitszeichen signalisieren, die das Geld formaljuristisch unabhängig von seinem Metallwert werden lassen sollten. ${ }^{12}$ Natürlich wirkte in der Realität des Marktgeschehens die Auffassung weiter, Gold und Silber seien Träger eines valor intrinsecus (ansonsten wäre die kaiserliche Zentrale aller Sorgen enthoben gewesen und hätte Papiergeld ausgeben können). Die Gesamtkonstellation implizierte, daß die Untertanen unter Androhung empfindlicher Strafen gezwungen wurden, daß kaiserliche Geld zu akzeptieren und für Markttransaktionen zu verwenden. Dies ist eine der Ursachen dafür, daß die prozessierende Verschlechterung

${ }^{9}$ Vgl. den Graph bei Depeyrot und Hollard 1987, a.a.O. (Anm. 8), 69: Die indizierte Menge des emittierten Silbers sank von einer Indexziffer von ca. 80 um 244 n. Chr. auf eine Indexziffer 10 um 275 n. Chr.

${ }^{10}$ Vgl. die Graphik von Depeyrot und Hollard 1987, a.a.O. (Anm. 8), 63.

${ }^{11}$ Depeyrot und Hollard 1987, a.a.O. (Anm. 8), 62f. Daß die Autoren einen ,brutalen' Rückgang des Münzaustoßes unter Aurelian konstatieren, ist wohl eine Fehleinschätzung, die darauf zurückzuführen ist, daß ihre Auswertung sich wesentlich auf Horte aus dem nördlichen (ehemaligen) Imperium Galliarum stützt (ibid. 59), wo sich der aurelianus zunächst nicht durchsetzte (vgl. Anm. 13). Vgl. jedoch zur Reduzierung der Offizinen unter Carus J.-P. Callu, La politique monétaire des empereurs romains de 238 à 311 (Paris 1969), 337.

12 Die grundlegende Studie ist K. Hasler, Studien zu Wesen und Wert des Geldes in der römischen Kaiserzeit von Augustus bis Severus Alexander (Bochum 1980), besonders 62ff. (,,pecunia als materia forma publica percussa"). 
des Geldes keinen unmittelbaren Zusammenbruch der Zirkulation nach sich zog. Im Gegenteil: Noch die aurelianische Reform des antoninianus vom Frühjahr 274 war unter fiskalischen Gesichtspunkten ein relativer Erfolg, denn die aureliani setzten sich zumindest im Osten als Verkehrmünzen vollkommen durch. ${ }^{13}$ Diese Erfolge der Herrscher, ihre Münzen in Zirkulation zu setzen, sind vor allem damit erklärt worden, daß die Untertanen die jeweils nur geringen (im einstelligen Prozentbereich liegenden) Feingehaltsreduktionen mit den zur Verfügung stehenden technischen Mitteln nicht hätten registrieren können. ${ }^{14}$ Auf der anderen Seite seien auch die Schwankungen des Feingehalts und des Gewichts innerhalb derselben Prägeperiode oder sogar Emission so bedeutend gewesen, daß die konkrete Metallverschlechterung oder -verbesserung von einer Emission zur folgenden für die Wirtschaftteilnehmer nicht fühlbar gewesen wäre. ${ }^{15}$ Soweit die kleinschrittigen Bewegungen von einer Emission zur folgenden betroffen sind, sind diese Beobachtungen durchaus zutreffend. Doch es ist vollkommen ausgeschlossen, daß den Untertanen des Imperiums der säkulare Trend der Edelmetallreduktion entgangen ist, d. h. nach menschlichem Ermessen bestand auch unter Aurelian noch eine Erinnerung daran, daß Silbermünzen einmal aus Silber hergestellt worden waren. ${ }^{16}$ Der hart sanktionierte Zwang, die gewissermaßen ,spartanisierte' Währung anzunehmen, ermöglichte dennoch über lange Zeitstrecken die formale Aufrechterhaltung der Zirkulation. ${ }^{17}$ Die inflationären Reaktionen erfolgten (soweit das Material zur Preisentwicklung dies erkennen läßt) in Schüben, ganz offenkundig in Form von Entladungen aufgestauten Vertrauensverlustes. ${ }^{18}$ Wenn es sich bei diesen Preisauftrieben lediglich um momentane Krisenindikatoren

${ }^{13}$ S. Estiot, ,Aureliana', Revue Numismatique 150 (1995), 50ff., 54f. Die Außerkraftsetzung der antoniniani gelang nicht auf dem Gebiet des ehemaligen gallischen ,Sonderreichs. ${ }^{6}$

${ }^{14}$ Hasler 1980, a.a.O. (Anm. 12), 125.

${ }^{15}$ K. Strobel, ,Geldwesen und Währungsgeschichte des Imperium Romanum im Spiegel der Entwicklung des 3. Jahrhunderts n. Chr.', ders. (Hrsg.), Die Ökonomie des Imperium Romanum, (St. Katharinen 2002), 86ff.; 96ff; 104ff.

${ }^{16}$ Vgl. Zosimos, Historia nova 1.61.3 und bspw. Cassius Dio 78(79).14.4 (zu Severus und Caracalla).

${ }^{17}$ Hasler 1980, a.a.O. (Anm. 12), 69f. (Annahmegebot); 84ff. (strafrechtliche Sanktionierung). Die Nichtannahme kaiserlichen Geldes (vultu principum signata moneta) war strafrechtlich der Fälschung gleichgestellt (Pseudo-Paulus, Sententiae 5.25.1). Zu den schweren (und regelmäßig verschärften) Strafen für das crimen falsi vgl. auch Th. Pekáry, ,Studien zur römischen Währungs- und Finanzgeschichte von 161 bis 235 n. Chr., Historia 8 (1959), 444ff., 459ff.

${ }_{18}$ Vgl. das Material bei Rathbone 1996, a.a.O. (Anm. 3); siehe auch Anm. 20. 
gehandelt hätte, wie wiederholt postuliert worden ist, ${ }^{19}$ dann hätten die Preise nach Beendigung der akuten Krisen wieder fallen müssen. Stattdessen sehen wir nachhaltige Anpassungsreaktionen, ${ }^{20}$ die latente Vertrauensverluste nun ökonomisch in Form von erhöhten Preisen sichtbar machten. - Das Argument, ,in Gold` ausgedrückt habe sich von Augustus bis Diokletian kein wesentlicher Preisauftrieb ergeben, ${ }^{21}$ geht an den ökonomischen Realitäten des Imperium Romanum vorbei: Weder zahlten die meisten Untertanen in Gold, noch garantierte die Regierung einen Goldstandard, zu dem das Markttransaktionsgeld in Relation gesetzt war.

Nun ist die Bestimmung der ökonomischen und sozialen Folgen der Metallmanipulationen eine äußerst problematische Aufgabe. Der säkulare Preisauftrieb war eine offenkundige Realität. ${ }^{22}$ Doch dürften sich regional und punktuell je nach der konkreten Situation sehr unterschiedliche Folgen der Geldverschlechterung ergeben haben. Beispielsweise waren die Quantitäten des zirkulierenden Geldes in aufeinander folgenden Zeitabschnitten und regional sehr unterschiedlich, ${ }^{23} \mathrm{~d}$. h. unter Umständen waren die Münzen nicht nur schlecht, sondern auch knapp (was etwa die verbreiteten Fälschungen in Peripherieregionen erklärt). ${ }^{24}$ Solche mit Geld unterversorgten Märkte haben offenkundig mit anderen Problemen zu kämpfen als überschwemmte Märkte.

Diesen Problemen kann hier nicht weiter nachgegangen werden. Betrachtet werden soll an dieser Stelle zunächst nur das Phänomen der ,Armut der kaiserlichen Zentrale,' die die Metallmanipulationen schließlich nicht aus Freude am wirtschaftspolitischen Experimentieren,

${ }^{19}$ Meißner 2000, a.a.O. (Anm. 3), 98; Rathbone 1996, a.a.O. (Anm. 3); Strobel 2002, a.a.O. (Anm. 15), $120 \mathrm{ff}$.

${ }^{20}$ Callu 1969, a.a.O. (Anm. 11), 394ff.; K.W. Harl, Coinage in the Roman Economy (Baltimore u.a. 1996), 280ff.; Rathbone 1996, a.a.O. (Anm. 3).

${ }^{21}$ Vgl. M. Corbier, 'Dévaluations et évolution des prix', Revue Numismatique, sér. 6, 27 (1985), 69ff.

${ }^{22}$ Vgl. Anm. 20. Ein Pfund Gold kostete zu Beginn des ersten Jahrhunderts 1000 Denare, im Jahr 300 betrug der Preis 60000 Denare (J. Lafurie, ,Réformes d'Aurélien et de Dioclétien', Revue Numismatique, sér. 6, 17 [1975], 73ff., 78). Viel weitergehende brutale Inflationssprünge erfolgten dann jedoch im 4. Jahrhundert: J.M. Carrié und A. Rousselle, L'Empire romain en mutation (Paris 1999), 568ff.

${ }^{23}$ Vgl. etwa zu den unterschiedlichen Konstellationen innerhalb Ägyptens Drexhage 1991, a.a.O. (Anm. 6), 450ff. Bemerkenswert ist das äußerst pessimistische Urteil dieses ausgezeichneten Kenners der Materie (z. B. 445: , [... ] in Zeiten großer Teuerung ist es für uns ein Rätsel, wie die Menschen [sc. Lohnarbeiter] überlebt haben können.").

${ }^{24}$ Vgl. bspw. M.R.-Alföldi, ,Die Gußformen und gegossenen „Fälschungen“ kaiserzeitlicher Münzen', Chiron 1 (1971), 351ff. 
sondern durch Not gezwungen vornahm. Für die Kaiser hatte ihre ,Verarmung' erhebliche Legitimitätseinbußen zur Folge: Das Leben eines Kaisers war den Soldaten schon seit Ende des zweiten Jahrhunderts nicht mehr viel wert. Eine geringfügige Enttäuschung über ein schwächer als erwartet ausgefallenes Donativ oder andere vergleichbar begrenzte Anlässe reichten als Motiv aus, damit ein Kaiser ohne Skrupel erschlagen wurde. Die historisch abgeleitet zentrale Aufgabe der Kaiser, die Militärklientel an sich zu binden, konnte nicht mehr erfüllt werden, wenn die Herrscher aufhörten, das redistributive Zentrum zu sein.

\section{Die Ursachen für die Verarmung der kaiserlichen Zentrale}

Wie ist es zu dieser Konstellation gekommen? Die römische Armee ist von Augustus bis Septimius Severus keineswegs dramatisch angewachsen: die Zahl der Legionen stieg von 25 auf 33 bei gleichzeitigem Zugewinn ausbeutbarer Provinzialböden und -bevölkerungen. Viel stärker zu Buche schlugen die Veränderungen in der Besoldungsstruktur. Allein die Hinzufügung eines vierten Stipendiums unter Domitian dürfte die Belastung der zentralen Kassen (in Verbindung mit der Erhöhung der Truppenzahlen unter Caligula und Vespasian) von (augusteisch) ca. 370 Millionen auf ca. 600 Millionen Sesterzen per annum vergrößert haben. ${ }^{25}$ Im zweiten Jahrhundert reduzierte die kaiserliche Regierung sukzessive die Deduktionen, die vom Sold für Ausrüstung und Verpflegung einbehalten wurden. ${ }^{26}$ Es folgten die nicht genau bezifferbaren, aber sehr beträchtlichen Solderhöhungen unter Septimius Severus, Caracalla und Maximinus, die - einer Schätzung zufolge - insgesamt auf ein Niveau von etwa $280 \%$ gegenüber dem augusteischen Niveau führten. ${ }^{27}$ Richard Duncan Jones ${ }^{28}$ hat wahrscheinlich gemacht, daß vor allem aufgrund

${ }^{25}$ Campbell 2002, a.a.O. (Anm. 2), 176.

${ }_{26}$ M.A. Speidel, 'Roman Army Pay Scales', Journal of Roman Studies 82 (1992), 87ff., 97.

${ }^{27}$ J. Jahn, ,Zur Entwicklung römischer Soldzahlungen von Augustus bis auf Diocletian', Studien zu den Fundmünzen der Antike 2 (1984), 53ff., 67. S. bspw. auch M.A. Speidel, Die römischen Schreibtafeln von Vindonissa (Brugg 1996), 64. Abweichende Schätzungen (vgl. die Diskussion in den genannten Beiträgen) bringen keine Veränderungen bezüglich der allgemeinen Größenordnungen.

${ }^{28}$ Structure and Scale in the Roman Economy (Cambridge u. a. 1990), 115, berechnet auf der Grundlage seiner Rekonstruktion der Angaben in den Beatty Panopolis-Papyri 12400 Rechendenare reguläre Zahlungserwartungen per annum einschließlich Donative für ,alares and probably for legionaries.' Daß präzise Kalkulationen auf der Basis der 
der Verstetigung der regelmäßig gezahlten Donative die Nettozahlungen in diocletianischer Zeit (nach vermutlich deutlicher Erhöhung der Truppenzahlen ${ }^{29}$ noch einmal erheblich über dem maximinischen Niveau lagen. Die Belastungen, die durch Solderhöhungen entstanden, erscheinen in der Tat als so erheblich, daß sie allein das Faktum der ,Verarmung der Zentrale‘ erklären könnten. Ein bestimmter Aspekt allerdings läßt das Verharren bei den Solderhöhungen als monokausaler Erklärung unbefriedigend erscheinen: Die Soldinflation war nur Teil der Dynamik, die die zentralen Kassen unter starken Druck setzte, und nicht der eigentlich bestimmende. Wenn das politisch-ökonomische Gesamtgefüge des Imperiums und seiner Umwelt stabil gewesen wäre, hätten die Herrscher die Notwendigkeit zu drastischen Solderhöhungen nicht empfunden. Die Aufstockung der Zahlungen war jedoch eine Reaktion auf die Legitimitätserosion, die oben skizziert wurde. Die Solderhöhungen lagen, soweit das absehbar ist, in zeitlicher Nähe zu Münzdevaluationen: ${ }^{30}$ Dies ist deutlich bei Domitian (der auf die neronischen und vespasianischen Devaluationen reagierte), wohl auch bei Caracalla und Maximinus, wobei sich, wie im dritten Jahrhundert generell, streiten läßt, ob im Einzelfall die Kaiser Münzverschlechterungen durch Solderhöhungen auszugleichen bestrebt waren oder auf die von ihnen verursachten Mehrausgaben mit Devaluationen reagierten. Wichtiger ist ohnehin, daß die Solderhöhungen in das Gesamtgefüge einer Bewegung gehörten, die die kaiserliche Zentrale einem immer stärkeren Ausgabendruck unterwarf. In diesem Kontext sind auch zu nennen die Baumaßnahmen im Bereich der militärischen Infrastruktur (einschließlich der Befestigung städtischer Kerne, die Lokalstaaten wohl häufig nicht mehr aus eigener Kraft bewältigen konnten). ${ }^{31}$

Beatty Panopolis-Papyri nicht möglich sind, unterstreicht R. Alston, 'Roman Military Pay from Caesar to Diocletian', Fournal of Roman Studies 84 (1994), $113 \mathrm{ff} ., 120$.

${ }^{29}$ Die Quellenlage ist problematisch. M.J. Nikasie, Treilight of Empire (Amsterdam 1998), 76, geht von einer Sollstärke von „mehr als 650000 Soldaten“ im vierten Jahrhundert aus und setzt 450000 als „Minimum“ der De-facto-Stärke. Überblick über die vorgeschlagenen Zahlen: Richardot 2001, a.a.O. (Anm. 4), 76. R. MacMullen, 'How big was the Roman Army?', Klio 62 (1980), 451ff. rechnet (452) mit 350,000-400,000 Soldaten bereits für die severische Armee.

${ }^{30}$ D.R. Walker, The Metrology of the Roman Silver Coinage. BAR Suppl. 40 (Oxford 1978), 3, 106ff;; tabellarische Übersicht: 138.

31 Vgl. bspw. zu Probus: G. Kreucher, Der Kaiser Marcus Aurelius Probus und seine Zeit. Historia Einzelschriften 174 (Stuttgart 2003), 219ff.; zu Diocletian: R. Rebuffat, ,Comme les moissons à la chaleur du soleil,' L'Africa Romana 6 (Sassari 1989), 113ff. 
Das entscheidende Moment, auf das die Reichsregierung keinen Einfluß hatte und das die Dynamik vorwärts trieb, war der militärische Druck auf die Grenzen des Reiches, oder besser: die Art und Weise, in der sich dieser Druck fühlbar machte, nämlich in zunehmender Weise an mehreren, häufig wechselnden Kriegschauplätzen zur gleichen Zeit. Das gesamte Konstrukt einer an den Reichsgrenzen aufgestellten Berufsarmee hätte in der Tat wohl stabil sein können, wenn die politisch-strategische Großkonstellation, mit der Augustus gerechnet hatte, stabil geblieben wäre (wogegen allerdings jede historische Wahrscheinlichkeit sprach). Augustus hatte große Verbände da massiert, wo zu seiner Lebenszeit größere Konflikte mit Nachbarn ausgetragen worden waren: am Rhein, am Euphrat und in Ägypten. Die Verlegung dieser Verbände war ein aufwendiges und vor allem kostspieliges Unterfangen, das, wenn es sich um langfristig im voraus geplante Zusammenziehung von Kampfverbänden an einem militärischen Brennpunkt handelte, vom fiskalisch-logistischen System des Imperiums gut bewältigt werden konnte. Die Überforderung setzte ein, wenn in schneller Folge mehrere Kampfverbände gebildet und über große Strecken verlegt werden mußten.

Nun ist dieses Faktum wiederholt konstatiert worden, in der jüngeren Zeit beispielsweise von Ramsay MacMullen ${ }^{32}$ und Fritz Mitthof. ${ }^{33}$ Ich möchte zu dem Gesagten einige Bemerkungen und Zahlen hinzufügen. Zunächst: Warum war eine Armee auf dem Marsch und im Krieg teurer als eine, die sich in ihren Standquartieren aufhielt? MacMullen hat die Transportkosten in den Mittelpunkt seiner Argumentation gerückt. ${ }^{34}$ Der Verweis auf Transportkosten ist plausibel, bedarf aber in seiner Pauschalität einer gewissen Relativierung. So waren nach den Schätzungen von David Breeze ${ }^{35}$ in Britannien zu Beginn des dritten Jahrhunderts zwischen ca. 47000 und 55000 Soldaten stationiert (für die ein Jahresbedarf von ca. 2,5-3 Millionen modii Getreide veranschlagt werden kann). Berücksichtigt man, daß die Soldaten in familienähnlichen Verhältnissen lebten, daß eine Vielzahl ${ }^{36}$ von Tieren (Pferde, Maultiere, Opfer- und Schlachtvieh) militärisch genutzt wurde und

32 'The Roman Emperors' Army Costs', Latomus 43 (1984), $571 \mathrm{ff} ., 576 \mathrm{f}$.

33 Annona Militaris. Die Heeresversorgung im spätantiken Ägypten (Firenze 2001).

${ }^{34}$ Roman Government's Response to Crisis (New Haven 1976), 105.

35 'Demand and Supply on the Northern Frontier', in R. Miket u.a., Hrsgg., Between and beyond the Walls (Edinburgh 1984), 264ff., 268.

${ }^{36}$ Nach Breeze 1984 a.a.O. (Anm. 35), 271, ist eine hohe fünfstellige Zahl für Britannien zu veranschlagen. 
daß außer Getreide zahlreiche Verbrauchsgüter benötigt wurden, ist deutlich, daß auch die Versorgung der ruhenden Einheiten erhebliche Transportleistungen erforderte. Auch die hochgradige Streuung der Quartiere machte die Versorgung der provinzialen exercitus zu einer anspruchsvollen Aufgabe.

Der ausschlaggebende Aspekt ist, daß die militärischen Einheiten im Laufe einer langen Stationierungsgeschichte ökonomisch und administrativ in ihren jeweiligen Heimatregionen ,verwurzelten.' Die entsprechenden Strukturen wurden regelmäßig gestört, wenn Einheiten aus ihren permanenten Stationierungskontexten herausgerissen und beweglichen Großverbänden zugeordnet wurden. Einzelmomente der ,Verwurzelung' sind in jüngeren Studien detailliert erforscht worden; einige der Ergebnisse seien kurz skizziert.

Militärische Einheiten bildeten eigene ökonomische Infrastrukturen (a) aus bzw. profitierten von sekundär durch die Militärpräsenz induzierten Strukturen ([b]; zwischen den beiden Polen existierte eine Skala von Übergängen). Zu den erstgenannten gehört das von Harald von Petrikovits sogenannte ,militärische Nutzland, ${ }^{‘ 37}$ das von Einheiten beispielsweise als Weideland (Tab. Vindol. 2,180) oder Holzreservoir genutzt werden oder an Pächter in Parzellen ausgegeben werden konnte. Zur militäreigenen ökonomischen Infrastruktur rechnen auch die den jeweiligen Einheiten zugeordneten und ihrer Leitung unterstehenden Werkstätten ( fabricae).$^{38}$ Lagerdörfer und Marketendersiedlungen waren eine Art Grenzfall, insofern sie zwar ,sekundär' an der Existenz von bestehenden Lagern ansetzten, ihre Entstehung, Lage und Gestaltung jedoch von der lokalen militärischen Kommandantur wesentlich beeinflußt wurde. ${ }^{39}$ Diese Strukturen sicherten den garnisonierten Einheiten eine berechenbare Zufuhr der jeweils produzierten oder gehandelten Güter.

${ }^{37}$ Vgl. H. von Petrikovits, ,Militärisches Nutzland in den Grenzprovinzen des römischen Reiches,' in Actes du VII congrès d'épigraphie greque et latine, 1977 (Bukarest 1979), 229-242. Zur Realisierung wirtschaftlicher Gefälle (reditus castelli) vgl. bspw. Tab. Vindol. 2,178; W. Eck, ,Die legio I Minervia. Militärische und zivile Aspekte ihrer Geschichte im 3. Jh. n. Chr.'; Y. Le Bohec, Hrsg., Les légions de Rome sous le Haut-Empire (Lyon 2000), 1,87ff., besonders 88.

${ }^{38}$ Vgl. bspw. Tab. Vindol. 2,155; Speidel 1996, a.a.O. (Anm. 27), 164ff.; M.P. Speidel, ,Zum Aufbau der Legion und zum Handwerk in Vindonissa', Roman Army Studies II (Stuttgart 1992), 56ff.

${ }^{39}$ C.R. Whittaker, 'Supplying the Roman Army. Evidence from Vindolanda', in P. Erdkamp, Hrsg., The Roman Army and the Economy (Amsterdam 2002), 204-235, $216 f$. 
Die Hauptmasse der Versorgungsgüter konnte jedoch nicht vom Militär selbst produziert werden und mußte extern bereitgestellt werden (b). Dieser Versorgungsbedarf militärischer Einheiten konnte in der Regel nicht aus dem unmittelbaren Stationierungsumland gedeckt werden. Aus diesem Grunde spielten sich innerhalb größerer Regionen Lieferwege und -modalitäten ein, die die Stetigkeit des Nachschubs gewährleisteten. Die Basiskonfiguration ist offenbar so beschaffen gewesen, daß die Kommandeure der jeweiligen Einheiten Liefergebote aussprechen (also auf Requirierungen zurückgreifen) konnten, ${ }^{40}$ darüber hinaus jedoch zusätzliche Güter, vor allem solche, die über den unmittelbaren Überlebensbedarf hinausgingen, auf dem freien Markt nachfragten, in der Regel wohl, indem sie Lieferkontrakte mit Privatunternehmern schlossen. ${ }^{41}$ Meist ist wohl auch für requirierte Güter eine Entschädigung, deren Höhe staatlich festgesetzt wurde, gezahlt worden. Fritz Mitthof hat den Vorgang der Requirierung (in diesem Fall von Gerste) anhand des sogenannten Damarion-Archives, ${ }^{42}$ einer Papyrusgruppe aus der Zeit des Commodus, dargestellt. Der Requirierungsbefehl, der die Versorgung einer bestimmten Reitereinheit mit Gerste sicherstellen sollte, wurde von der Provinzleitung publiziert und sah eine anteilige Beteiligung der Dörfer der gesamten Provinz vor. Entsprechend ihrer Belastung wurde den Kontribuenten aus dem Provinzialfiscus eine staatlich festgesetzte Vergütung bezahlt. ${ }^{43}$ Der Bericht läßt sich nur hinsichtlich der Basisstruktur, nicht in seinen Details verallgemeinern, worauf Mitthof in seiner grundlegenden Untersuchung, die auch das Quellenmaterial außerhalb Ägyptens ausführlich einbezieht, nachdrücklich hingewiesen hat. Tatsächlich lassen sich für einzelne Proviantgüter (z. B. Weizen, Futtergerste, Spreu, Öl, Wein usw.) jeweils voneinander abweichende Liefer- und Requirierungstypen ermitteln, die auch regional und historisch-chronologisch bedeutende Unterschiede aufweisen. Aus diesem Grunde bieten die Quellen (wie etwa die Ostraka aus Bu Njem, die Papyri aus Dura Europos, die Wachstafeln aus Vindonissa, Tolsum oder Vindolanda) ein vielgestaltiges Bild, in dem direkte Requirierung durch

${ }^{40}$ Vgl. bspw. Whittaker 2002, a.a.O. (Anm. 39), 228f.; P. Erdkamp, 'The Corn Supply of the Roman Army during the Principate', ders., Hrsg., The Roman Army and the Economy (Amsterdam 2002), 56ff.

${ }^{41}$ C.E.P. Adams, 'Supplying the Roman Army: O. Petr. 245', Zeitschrift fir Papyrologie und Epigraphik 109 (1995), $119 f f$.

${ }^{42}$ Verzeichnis der Editionen und ausführliche Darstellung bei Mitthof 2001 a.a.O. (Anm. 33), 2, 314ff.

${ }^{43}$ Mitthof 2001 a.a.O. (Anm. 33), 1, 43. 
Militärpersonal, ${ }^{44}$ Lieferungen durch tributäre Bevölkerungsgruppen (O. Bu Njem 76ff;; Tacitus, vita Agricolae 19.4), Ankauf in privater Initiative militärischen oder militärnahen Personals (FIRA 3,137), Lieferung durch private Unternehmer ${ }^{45}$ und Übersendung von militäreigenem Nutzland oder praedia fiscalia (P. Dura 64) nebeneinander belegt sind.

In einer Reihe archäologisch orientierter Studien ist gezeigt worden, daß sich in Regionen, die durch Militärpräsenz geprägt waren, die Produktions- und Infrastruktur auf die Versorgungsbedürfnisse der betreffenden militärischen Einheiten ausrichtete. Genannt seien hier die Arbeiten von Charles Whittaker, ${ }^{46}$ Paul Middleton ${ }^{47}$ und Maureen Carroll. ${ }^{48}$ Als typisch tritt hervor, daß sich partiell spezialisierte Versorgungszonen bildeten, aus denen bestimmte Güter oder Gütergruppen an die militärischen Konsumenten geliefert wurden. So hat etwa Carroll unter Heranziehung des keramischen Fundmaterials des niedergermanischen Flottenhafens (Alteburg) solche Versorgungsregionen rekonstruiert (Getreide aus dem belgischen Lößgürtel, Fleisch und Tierhäute aus dem friesischen Gebiet, Fisch von der friesischen Küste, Salz „,von der Küste über das Rhein-Schelde-Delta“)..$^{49}$ Middleton (1979, a.a.O. [Anm. 47]) hatte ähnliche Zuordnungen unter anderem innerhalb des gallischen Raumes rekonstruiert und betont, daß die Versorgungsleistungen durch eine komplexe Verschränkung von jeweils lokalen, speziellen Bedürfnissen angepaßten Arrangements (vergleichbar den von Mitthof für Ägypten nachgewiesenen speziellen Versorgungswegen für jeweils einzelne Güter), großräumiger Requirierung und einem gewissermaBen ,parasitär ${ }^{6}$ ansetzenden privatwirtschaftlichem Handel bewältigt wurden. Auch wo nicht von ,Spezialisierung von Versorgungsregionen“ gesprochen werden kann, ist eine starke Strukturierung von Regionen entsprechend den Versorgungsbedürfnissen des Militärs zu beobachten, etwa in der Ausdifferenzierung der latifundistischen Zone im Süden Britanniens als ,Versorgungsdistrikt' der Militärzone am Hadrians- bzw.

\footnotetext{
${ }^{44}$ Quellen bei Whittaker 2002, a.a.O. (Anm. 39), 211, $228 f f$.

45 Vgl. die dokumentarischen Belege bei Adams 1995, a.a.O. (Anm. 41), 124, note 32 .

${ }^{46}$ 2002, a.a.O. (Anm. 39).

47 'Army Supply in Roman Gaul: An Hypothesis for Roman Britain', in Barry C. Burnham u.a., Hrsgg., Invasion and Response. The Case of Roman Britain (London 1979), $81 \mathrm{ff}$.

48 'Supplying the Roman Fleet: Native Belgic, Frisian and Germanic Pottery from Cologne', Fournal of Roman Archaeology 14 (2001), $311 \mathrm{ff}$.

49 2001, a.a.O. (Anm. 48), 320f.; C.R. Whittaker, Frontiers of the Roman Empire (Baltimore u.a. 1994), $118 f f$.
} 
Antoninuswall. ${ }^{50} \mathrm{Zu}$ beachten sind in diesem Zusammenhang auch die Handels- und Versorgungssymbiosen, die sich zwischen den Militärzonen und den Ethnien jenseits der Militärgrenzen herauszubilden pflegten. ${ }^{51}$

Die skizzierten ökonomischen und administrativen Bindungen von Einheiten an ihre Stationierungsregion wurden zerrissen, wenn eine Einheit (oder Abteilung der Einheit) den Marschbefehl erhielt und fern von ihrem Heimatmilieu versorgt werden mußte. Das ist gewissermaßen der negative kostentreibende Effekt. Auf der anderen, positiven Seite waren noch eine Reihe von Faktoren wirksam, die die Kosten der Verlegungen in die Höhe trieben. Zunächst änderte sich die fiskalische Struktur der Versorgung. Fritz Mitthof beschreibt die Vorgänge für Ägypten (Ende des zweiten Jahrhunderts) mit folgenden Worten:

Es handelte sich um einen auf landesweiter Ebene durchgeführten Zwangseinkauf von Lebensmitteln aller Art, wobei das vom Staat angeforderte Gesamtaufkommen mittels Repartition zunächst auf die Gaue, sodann auf die Dörfer umverteilt wurde. Die Staatskasse streckte den Lokalbehörden das zum Ankauf benötigte Geld vor, damit den Kontribuenten der Gegenwert des requirierten Gutes unmittelbar bei dessen Ablieferung erstattet werden konnte. ${ }^{52}$

In wesentlichen und den hier ausschlaggebenden Elementen waren die Modalitäten in anderen Provinzen ähnlich (natürlich vollzog sich die Einbindung der munizipalen Ebene in anderer Weise). In aller Regel zahlte eine von der kaiserlichen Zentrale kontrollierte Kasse einen administrativ fixierten Preis für die gelieferten Güter, der zwar unter den Marktpreisen lag, aber sich dennoch an Marktpreisen orientierte. Sowohl naheliegende ökonomische Überlegungen als auch Quellen verschiedener Gattungen ${ }^{53}$ führen darauf, daß die Anwesenheit großer Heeresverbände die Lebensmittelpreise deutlich in die Höhe trieb.

${ }^{50}$ Middleton 1979, a.a.O. (Anm. 47), 91ff.; Whittaker 1994, a.a.O. (Anm. 49), 107ff;; 128.

${ }_{51}$ Whittaker 1994, a.a.O. (Anm. 49), 98ff.

${ }_{52}$ Mitthof 2001, a.a.O. (Anm. 33), 1,53 (nach PSI 683). ,Zwangseinkäufe‘ waren natürlich auch ein Element der Versorgung ruhender Verbände. Aber erstens nur eines unter mehreren, zweitens waren die ziehenden Verbände in der Regel zusätzlich zu den ruhenden Verbänden zu versorgen, drittens waren die Transportprobleme viel gravierender, eben weil die Adressaten der Lieferungen sich bewegten. Die Zwangseinkäufe fanden schließlich nicht nur ,landesweit,' sondern provinzübergreifend statt.

${ }^{53}$ R. Ziegler, Kaiser, Heer und städtisches Geld (Wien 1993), 139ff., besonders 142; Mitthof 2001, a.a.O. (Anm. 33), 1, 80f.; M. Giacchero, Edictum Diocletiani et Collegarum de pretiis rerum venalium (Genova 1974), $134 \mathrm{ff}$. 
Die Heeresleitung mußte zu einem bestimmten Grad auf den Preisauftrieb reagieren, wenn sie keine Güterflucht provozieren wollte. Da die Nachfrage der Zentrale bei größeren Truppenbewegungen intensiv und reichsweit (vgl. etwa Herodian, Historia post Marcum 6.2.3; 7.8) einsetzte, mußten die Folgen für die Märkte der ganzen Ökumene spürbar sein.

Es war allerdings nicht nur diese ,fundamental reliance on cash, ${ }^{64}$ die die Kosten für die kaiserliche Zentrale in Kriegszeiten in die Höhe trieb, sondern unter anderem auch das gut belegte Faktum, daß die Kopfstärke der Einheiten im Kriegsfall unter Umständen erheblich erhöht wurde. Generell fallen größere Aushebungen der Kaiserzeit in Kriegsphasen (oder unmittelbar in die Zeit nach Kriegsende, wenn Verluste auszugleichen waren). ${ }^{55}$ Rachel Feig Vishnia ${ }^{56}$ hat darüber hinaus in ihrer Arbeit über die lixae - die Schattenarmee der Legionen - darauf aufmerksam gemacht, daß marschierende Verbände häufig zahlreiche Hilfskräfte auf Kontraktbasis anwarben, die in verschiedenen Bereichen, speziell der Bewachung und sonstigen, Versorgung' Kriegsgefangener eingesetzt wurden und die die De-facto-Stärke der Verbände unter Umständen nahezu verdoppeln konnten. In diesem Zusammenhang kann auch die Beobachtung Michael P. Speidels angeführt werden, der zufolge im dritten Jahrhundert (in Kriegsphasen) zunehmend Söldner zur Verstärkung regulärer Verbände angeworben wurden. ${ }^{57}$

\section{Zur Korrelation von militärischer Situation und kaiserlicher Kassenlage}

Es liegt nahe, eine Statistik der Kriege des Imperium Romanum zu betrachten, wenn man die Ursachen des, langsamen Zusammenbruchs ${ }^{6}$ des augusteischen fiskalischen Systems genauer benennen möchte. Dies ist natürlich keine originelle Beobachtung; und Zusammenstellungen von Daten sind bereits in instruktiver Weise vorgelegt worden, namentlich

\footnotetext{
${ }^{54}$ J.P. Roth, The Logistics of the Roman Army at War (Leiden u.a. 1999), 238.

55 MacMullen 1980, a.a.O. (Anm. 29), 453.

56 'The Shadow Army - the lixae and the Roman Legions', Zeitschrift fir Papyrologie und Epigraphik 139 (2002), $265 \mathrm{ff}$.

57 'The Rise of the Mercenaries in the Third Century', Roman Army Studies II (Stuttgart 1992), $71 \mathrm{ff}$.
} 
von David R. Walker ${ }^{58}$ (und jüngst von Yann Le Bohec). ${ }^{59}$ Jean-Pierre Bost $^{60}$ hat allerdings darauf hingewiesen, daß sich eine vollkommen eindeutige Korrelation von Münzausstoß, Devaluation und Kriegsintensität aus dem vorhandenen Material nicht ablesen läßt. Dies wäre auch überraschend: Es spielten zu viele Faktoren zusammen, die das Wirken eindimensionaler Kausalitätsverhältnisse verhinderten. Schon aus diesem Grund dürfen keine überzogenen Erwartungen an die Auswertung von Zahlenmaterial gestellt werden, das zudem aus einem lückenhaften Quellenmaterial gewonnen ist. Bezüglich der Auswertung dieses Materials soll hier nur im Anschluß an das oben Ausgeführte ein Interpretationsvorschlag gemacht werden. In einer umgehend noch näher zu erläuternden Tabelle habe ich einige Daten der römischen Militärgeschichte der ersten drei Jahrhunderte der römischen Kaiserzeit ausgewertet (Tabelle I). Bereits ein erster Blick auf diese Tabelle zeigt, daß die Verlegungsintensität von Truppenverbänden auch im ersten Jahrhundert n. Chr. recht hoch gewesen ist, so daß die oben gewählte Fokussierung auf Truppenbewegungen (im Kontext der Kostendynamik) fragwürdig erscheinen könnte. Nach meinem Dafürhalten ist jedoch die Auswertung der Truppenverlegungen mit der Betrachtung anderer typologischer Momente der römischen Kriege zu kombinieren, um den Sinn dieses Ansatzes richtig hervortreten zu lassen.

Das Imperium verfügte über ausreichende Ressourcen, um problemlos größere Verbände aus den jeweiligen Heeresbezirken (Britannien, Rheinprovinzen, Donauprovinzen, Anatolien und Syrien/Palästina, Ägypten) zusammenzuziehen und an einer Front zu konzentrieren. Dies war gewissermaßen der im augusteischen System einkalkulierte Normalfall einer dynamischen Aktivierung. Wenn im (systemimmanent betrachtet) günstigen Fall eine lange Zeitspanne für die logistische Vorbereitung zur Verfügung stand, wie beispielsweise für den von Domitius Corbulo an der Ostgrenze organisierten Aufmarsch, der über vier Jahre hinweg (54-58) durchgeführt wurde, stellte dies zwar eine erhebliche Belastung dar, die jedoch ohne Überstrapazierung der fiskalischen Kräfte bewältigt werden konnte. Die Regenerationsfähigkeit des fiskalischen Systems war

\footnotetext{
581978 a.a.O. (Anm. 30), 3,106ff.

59 'Les aspects militaires de la crise du III' siècle', ders. u.a., Hrsgg., L'armée romaine de Dioclétien à Valentinien I ${ }^{e r}$ (Lyon 2004), 10ff. (vgl. besonders die Tabelle 11f.).

60 'Guerre et finances, de Marc Aurèle à Maximin (161-238)', in J. Andreau, u.a., Hrsgg., Économie antique. La guerre dans les economies antiques (Saint-Bertrand-de-Comminges 2000), 399ff.
} 
Tabelle 1

\begin{tabular}{|c|c|c|c|c|c|c|c|}
\hline Jahrzehnt & $\begin{array}{l}\text { Jahre mit } \\
\text { kriegsbedingten } \\
\text { Verlegungen } \\
\text { von Legionen } \\
\text { zwischen } \\
\text { Armeebezirken }\end{array}$ & $\begin{array}{l}\text { Verlegungs- } \\
\text { intensität }\end{array}$ & $\begin{array}{l}\text { jeweils } \\
\text { betroffene } \\
\text { Fronten/ } \\
\text { Armeebezirke }\end{array}$ & $\begin{array}{l}\text { logistische } \\
\text { Vorbe- } \\
\text { reitung }\end{array}$ & $\begin{array}{l}\text { fiskalische } \\
\text { Erholung }^{1}\end{array}$ & $\begin{array}{l}\text { nachhaltiger } \\
\text { Ressourcen- } \\
\text { gewinn }\end{array}$ & $\begin{array}{l}\text { Legions- } \\
\text { dislokationen } \\
\text { aufgrund von } \\
\text { Bürgerkrieg }\end{array}$ \\
\hline $14-20^{2}$ & 3 & ++ & 1 & +++ & ++ & 0 & \\
\hline $21-30$ & 4 & + & 1 & +++ & +++ & 0 & \\
\hline $31-40$ & 1 & +++ & 1 & +++ & $(+)$ & 0 & \\
\hline $41-50$ & 8 & ++ & 1 & +++ & + & 0 & \\
\hline $51-60$ & 7 & +++ & 1 & +++ & - & 0 & \\
\hline $61-70$ & 10 & $++/+++$ & $1: 4 \mathrm{~J} . / 3: 6 \mathrm{~J} .^{3}$ & $\begin{array}{l}+++ \\
-\end{array}$ & -- & -- & $\begin{array}{l}+++ \\
\text { (3 Jahre) }\end{array}$ \\
\hline $71-80$ & 4 & ++ & 2:2J./3:2J. & +++ & +++ & + & $+(1 \mathrm{~J})$. \\
\hline $81-90$ & 8 & +++ & 1:6J./2:2J. & +++ & $+/(+)$ & -- & \\
\hline $91-100$ & 6 & +++ & 1 & +++ & $(++)$ & $-(+)$ & \\
\hline $101-110$ & 6 & +++ & 1 & +++ & $(+++)$ & +++ & \\
\hline $111-120$ & 5 & +++ & 1 & +++ & +++ & - & \\
\hline $121-130$ & 1 & + & 1 & +++ & +++ & - & \\
\hline $131-140$ & 6 & + & 1 & +++ & +++ & -- & \\
\hline $141-150$ & 2 & + & 1 & +++ & +++ & - & \\
\hline $151-160$ & 2 & + & 1 & +++ & +++ & - & \\
\hline $161-170$ & 10 & +++ & 1:7J./2:3J. & -- & --- & - & \\
\hline $171-180$ & 10 & +++ & 1:8J./2:2J. & - & --- & -- & $++(2 \mathrm{~J})$. \\
\hline $181-190$ & 0 & & & & +++ & & \\
\hline $191-200$ & 8 & +++ & 1:5J./2:3J. & + & -- & - & $+++(3 \mathrm{~J})$. \\
\hline $201-210$ & 7 & ++ & 1 & +++ & $(+)$ & -- & \\
\hline $211-220$ & 8 & +++ & 1 & +++ & $-/(++)$ & $-/--$ & $+(2 \mathrm{~J})$. \\
\hline $221-230$ & 0 & & & & +++ & 0 & \\
\hline $231-240$ & 10 & +++ & 1:5J./2:5J. & $+/--$ & --- & -- & $+(3 \mathrm{~J})$. \\
\hline $241-250$ & 10 & +++ & 1:9J./2:1J. & ++ & -- & - & \\
\hline $251-260$ & 10 & +++ & $\begin{array}{l}\text { 1:1J./2:2J./ } \\
3: 7 \mathrm{~J} .\end{array}$ & --- & --- & -- & $+++(4 \mathrm{~J})$. \\
\hline $261-270$ & 9 & +++ & 2:7J./3:2J. & --- & --- & -- & $+++(5 \mathrm{~J})$. \\
\hline $271-280$ & 8 & +++ & $\begin{array}{l}1: 2 \mathrm{~J} . / 2: 4 \mathrm{~J} . \\
3: 2 \mathrm{~J} .\end{array}$ & --- & --- & --- & $+++(4 \mathrm{~J})$. \\
\hline 281-285 & 3 & +++ & 1:2J./3:1J. & $+/-$ & + & -- & $+(1 \mathrm{~J})$. \\
\hline
\end{tabular}

1 Wenn die Erholungsphase erst in das Folgejahrzehnt fällt, ist dies mit Klammern $(+++)$ ausgedrückt.

2 Die Einträge für diesen Zeitraum sind zu lesen: Vier Jahre mit mittlerer Verlegungsintensität; ein Frontbereich war als Zielgebiet betroffen; die logistische Vorbereitungszeit war hoch, die anschließende Erholungsphase von mittlerer Länge. Ein nennenswerter Ressourcengewinn war nicht zu verzeichnen.

${ }^{3}$ Lies: Eine Front war vier Jahre lang Zielgebiet von Truppenverlegungen (bzw. Herkunftsgebiet von Rückverlegungen); drei Fronten über sechs Jahre hinweg. 
auf der anderen Seite erheblich: Wenn ihm nur einige Jahre der ,Ruhe, d. h. Abwesenheit von größeren Truppenverlegungen, gegönnt wurden, gewann es die finanziellen Kräfte für neue militärische Abenteuer sehr schnell zurück. Nachdem sich beispielsweise das Imperium, trotz des (angeblich von Commodus verschuldeten) finanziellen Notstands ${ }^{61}$ vier Jahre Bürgerkrieg und anschließend einige Aggressionen ${ }^{62}$ gegen östliche Nachbarn geleistet hatte, genügte eine Atempause von 202-207, bevor neue große Aufmärsche (in Britannien 208-211; Rhein- und Donau 213/14 und im Nahen Osten 214-218) und erhebliche Solderhöhungen wieder als finanzierbar empfunden wurden.

Um diesem Umstand Rechnung zu tragen, ist in der tabellarischen Zusammenstellung die fiskalische Regeneration und die logistische Vorbereitung mitberücksichtigt worden. Die logistische Vorbereitung ist natürlich nicht nur unter zeitlichem Aspekt zu betrachten; wichtig ist vor allem auch, ob der Aufmarsch auf Initiative der römischen Zentrale stattfand, oder ob die Gegner das Gesetz des Handelns diktierten. Besonders schwer fiel ins Gewicht, wenn in einer Konstellation, in der bereits massiv Verbände an bestimmten Frontabschnitten konzentriert waren, Verlegungen, schlimmstenfalls in schneller Folge an weit auseinander liegende Fronten, improvisiert werden mußten. Es ist daher natürlich zu beachten, ob mehrere Kriege an mehreren Schauplätzen gleichzeitig geführt wurden und welche Regenerationspausen zwischen den Kriegsagglomerationen lagen. Natürlich ist auch von Bedeutung, ob ein Krieg erfolgreich geführt wurde oder nicht, wobei unter der hier gewählten Perspektive der fiskalische Aspekt ausschlaggebend ist. Unter systemimmanenten Gesichtspunkten waren Trajans Dakerkriege aufgrund der Eroberung des Königsschatzes und der Erschließung der transsilvanischen Erzminen ein Erfolg; die Siege Aurelians waren dagegen fiskalisch betrachtet Niederlagen für das imperiale System.

Die angefügte Tabelle gibt nur grobe Näherungswerte. Dies ist einerseits mit dem zur Verfügung stehenden Raum zu begründen: Eine akkurate Ausbreitung des Materials würde mindestens ein Buch, wenn nicht mehrere Bände in Anspruch nehmen. Andererseits existiert noch

${ }^{61}$ Die von Cassius Dio (Historia Romana 73[74].5.4; vgl. 73[74].8.3) erwähnten eine Million Sesterzen, über die das ,basileion' nach Commodus' Tod noch verfügt habe, betreffen vermutlich nur die in Rom unmittelbar verfügbaren Gelder - und etwa nicht die Provinzialfisci, die selbstverständlich den ,zentralen Kassen` zugeordnet waren.

${ }^{62} \mathrm{Da}$ Severus sich genötigt sah, die neugewonnene Provinz Mesopotamia mit zwei neuen Legionen zu sichern, dürfte es sich bei den Erfolgen der Feldzüge von 195 und 198/99 im wesentlichen um Zuschußgeschäfte gehandelt haben. 
keine systematische Durcharbeitung der Quellen. Daher ist mit der rudimentären, hier gegebenen Tabelle ein Desiderat verbunden: Die Kriege des Imperiums müßten unter Auswertung aller zur Verfügung stehenden literarischen, epigraphischen, numismatischen, papyrologischen und archäologischen Quellen unter den oben genannten Gesichtspunkten (Quantität der Truppenverlegungen, fiskalische Belastung, Ergebnisse u. a.) untersucht werden, um ein präzises Bild von der fiskalischen Entwicklung des Imperiums und damit seines inneren Lebensgesetzes zu erhalten. In einer Hinsicht wäre allerdings auch eine sehr sorgfältige ,Statistik' aller Voraussicht nach stark verzerrt: Der Rückgang vor allem epigraphischer und papyrologischer Quellen seit der Mitte des dritten Jahrhunderts läßt eine befriedigende Rekonstruktion der Truppenbewegungen nicht mehr zu, so daß Schätzungen ein sehr breiter Raum gewährt werden muß. Imponderabilien gibt es ohnehin in groBer Zahl: Selbst die Schätzungen zu dem wahrscheinlich am besten dokumentierten Aufmarsch (im Vorfeld des ersten Dakerkriegs Trajans) differieren von ca. $100000^{63}$ bis zu ,nicht unter 200000 Mann. “64 Die spezifischen Probleme können hier, wie gesagt, nicht diskutiert werden. Die untenstehende Tabelle hat ihr Fundament in dem legio-Artikel Emil Ritterlings. ${ }^{65}$ Ergänzendes Material ist unter anderem aus Robert Saxers Studien zu den Vexillationen, ${ }^{66}$ John Spauls ${ }^{67}$ Arbeit zu den Auxiliarien und aus den von Yann Le Bohec edierten Bänden über die Legionen der römischen Kaiserzeit herangezogen worden. ${ }^{68}$

Zur Erläuterung und Interpretation ist nun Folgendes zu bemerken. Die augusteische Epoche, in der das System erst etabliert wurde, ist nicht berücksichtigt worden. Die erste Spalte gibt die Jahre eines Jahrzehntes, in denen größere Truppenverlegungen stattfanden. Damit

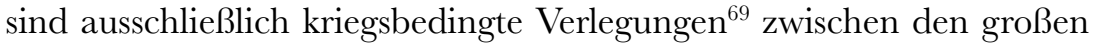
Stationierungsbezirken (Britannien, Rheinprovinzen, Donauprovinzen, Anatolien/Syrien-Palästina, Ägypten, Africa) und nicht innerhalb der

\footnotetext{
${ }^{63}$ J. Bennett, Trajan. Optimus Princeps, A Life and Times (London u.a. 1997), 89.

${ }^{64}$ E. Ritterling, 'legio', Realencyclopädie 12,1 (1924), $1211 \mathrm{ff.}, 1282$.

65 1924, a.a.O. (Anm. 64) und Realencyclopädie 12,2 (1925), 1330ff.

${ }^{66}$ Untersuchungen zu den Vexillationen des römischen Kaiserheeres von Augustus bis Diokletian (Köln u.a. 1967).

${ }^{67}$ Cohors. BAR International Series 841 (Oxford 2000).

${ }^{68}$ Les légions de Rome sous le Haut-Empire, 3 Bde. (Lyon 2000ff.).

${ }^{69}$ Wozu auch bloße Aufmärsche (wie etwa diejenigen, die Nero $64 \mathrm{n}$. Chr. für seine geplanten Axum- und Kaukasusfeldzüge anordnete) gerechnet worden sind, die mit der Absicht, einen Krieg zu führen, durchgeführt wurden.
} 
genannten Bezirke gemeint. Selbstverständlich sind ,Verlegungsjahre‘ nicht gleichzusetzen mit Kriegsjahren; dieser Umstand macht die Schätzung jedoch häufig sehr schwierig: Der Beginn eines Aufmarsches und die ,Abwicklung ${ }^{6}$ eines Invasionsheeres waren unspektakulär und sind daher häufig ganz unzureichend dokumentiert. In vielen Fällen sind daher nur Abwägungen möglich. In der zweiten Spalte ist versucht worden, die quantitative Intensität der Truppenbewegungen zu bewerten. Wie bereits erwähnt, ist der Spielraum für Schätzungen hier sehr groß: Die epigraphischen Belege lassen in aller Regel nur den Schluß auf Untergrenzen (bezüglich der betroffenen Einheiten oder Abteilungen von Einheiten) zu, während die antiken Autoren zu Übertreibungen zu neigen scheinen. Die Symbole versuchen eine ungefähre Einordnung zu geben. Wenn nur eine Legion (oder Vexillationen einer Legion) aus ihrem Bezugsraum verlegt wurde (wie beispielsweise $20 \mathrm{n}$. Chr. die legio IX Hispana aus Pannonien zur Repression des Tacfarinas-Aufstandes ${ }^{70}$ entspricht dies einem Pluszeichen. ${ }^{71}$ Ein Aufmarsch, für den Verbände aus mehreren Heeresabschnitten mobilisiert und zusammengefaßt wurden (wie $39 \mathrm{n}$. Chr. in den Rheinprovinzen) ${ }^{72}$ ist mit +++ taxiert worden. In der Spalte ,Fronten/Armeebezirke“ ist die Zahl der Zielgebiete größerer Truppenverlegungen angegeben worden.

Die Symbolik der drei folgenden Spalten gehorcht derselben Logik wie die Symbole des Abschnitts ,Verlegungsintensität.' Bei der Bewertung beispielsweise der logistischen Vorbereitung sind nicht nur die zeitliche Quantität (Anzahl der Jahre), sondern auch qualitative Umstände berücksichtigt worden. So stand für die Aufmärsche, die Nero 64 (Axum- und Kaukasus-Feldzug) befahl, zwar eine größere Planungszeit zur Verfügung, aber erstens war bereits ein großer Heeresverband an der Ostgrenze zu versorgen und (zweitens) nötigte der Jüdische Aufstand zum Umdirigieren und Improvisieren, so daß die fiskalische Gesamtbewertung insgesamt für diese Operationen negativ ausfällt (nicht zufällig beginnt mit Nero die Geschichte der Manipulationen des augusteischen Münzsystems). Bei der Beurteilung des Ressourcengewinns ist versucht worden, eventuelle Territorialgewinne und Kriegsbeute mit den Kosten der Operationen in Relation zu setzen (vgl. beispielsweise die Überlegung Anm. 62 zu Septimius Severus). Bürgerkriegsjahre, die

\footnotetext{
70 Tacitus, Annales 3.9; 4.23 .

71 Größere Konflikte, die im wesentlichen mit den Kräften eines Provinzexercitus bestritten wurden, sind in der Tabelle nicht verzeichnet.

${ }^{72}$ Quellen: Ritterling 1924, a.a.O. (Anm. 64), 1248.
} 
gewissermaßen eine Verdoppelung der logistischen Kosten implizierten und Verluste für das Imperium garantierten, sind in einer gesonderten Spalte geführt worden. Die Angaben der Spalten müssen miteinander in Kombination gesehen werden. Ausschlaggebend ist, über wieviel Jahre hinweg welche Verlegungsintensität (Zahl der beteiligten Verbände) herrschte und wieviel Grenzabschnitte zur (etwa) gleichen Zeit berücksichtigt werden mußten.

Wie bereits betont, befinden wir uns mit diesen Überlegungen in einem Bereich zahlreicher Konjekturen und subjektiver Einschätzungen. Die Tabelle beinhaltet im wesentlichen eine Aufforderung zur Ausarbeitung viribus unitis. Doch soviel läßt sich bei allen Kautelen doch aus den Daten ableiten: Es war nicht die Verlegungsintensität allein für sich genommen, die das fiskalisch-militärische System über seine Leistungsfähigkeit strapazierte, sondern eine Kombination aus verschiedene Faktoren: Nach einer längeren Erholungsphase in hadrianisch-antoninischer Zeit war das Imperium gezwungen, in immer kürzeren Abständen Kriege an mehreren Kriegsschauplätzen zugleich zu führen. Diese Tendenz verstärkte sich dramatisch seit 231 n. Chr. Damit war eine hohe und zunehmend improvisierte Verlegungsaktivität verbunden. Improvisation mußte die Kosten erhöhen, weil logistische Vorbereitungszeiten fehlten, wodurch der Zwang, Waren durch aktive Nachfrage zu dem Verband zu ziehen, sich vergrößerte. ${ }^{73}$ Dem steigenden Ausgabendruck stand gegenüber, daß ausreichende Regenerierungszeiten für die zentralen Kassen nicht mehr zur Verfügung standen. Hinzukam, daß die Kriege des dritten Jahrhunderts (nach den severischen Partherkriegen) nur noch Geld kosteten und kaum noch Gewinne brachten. Dieses Ursachengefüge zwang das fiskalische System (langsam, aber unerbittlich) in die Knie und verursachte die dauerhafte, Verarmung' der kaiserlichen Zentrale.

Die Reformen des vierten Jahrhunderts brachten noch einmal ein retardierendes Moment in die Entwicklung. Die Regularisierung der annona, die Verkleinerung der Legionen (d. h. die Behandlung der vexillatio als Normaltyp), die Schaffung des ,spätantiken Bewegungsheeres' und die Erschließung und konsequente Ausschöpfung neuer Steuerquellen (etwa des chrysargyron) sind Ausdruck dafür, daß die Reichsleitung den Ausnahmezustand des dritten Jahrhunderts als dauerhaft akzeptieren

73 Diokletian hat dies im edictum de pretiis anschaulich beklagt; vgl. Giacchero 1974, a.a.O. (Anm. 53), 134ff. 
mußte und entsprechend reagierte. Doch den Teufelskreis von militärischer Defensive, Verarmung der Zentrale und Legitimitätserosion konnten die aufeinander folgenden Regierungen nur noch verlangsamen, nicht mehr anhalten. 